\title{
EDUCACIÓN FÍSICA, DEPORTE, RECREACIÓN Y POLÍTICAS PÚBLICAS EN AMÉRICA LATINA
}

\author{
Alejo Levoratti ${ }^{1}$ \\ Juan Manuel Carreño Cardozo ${ }^{2}$
}

Esta sección central reúne una serie de producciones desarrolladas en el campo de las Ciencias Sociales que analizan la incorporación de la educación física, el deporte y las distintas prácticas corporales en diferentes políticas públicas en varios países de la región (Argentina, Brasil, Colombia, Uruguay). Investigadores provenientes de distintas disciplinas -antropología, ciencias de la comunicación, educación, educación física, filosofía y sociología- presentan una pluralidad de enfoques y perspectivas teóricas que, estudiándolas de forma articulada brindan un amplio panorama para la discusión del tema de esta sección central.

Además, esta edición de la revista Lúdica Pedagógica cuenta, por primera vez, con colaboración internacional para la dirección de la sección central, situación que refleja un avance importante en la interacción académica que los campos de la educación física, la recreación y el deporte necesitan en la región. En este sentido, es notable el desarrollo de propuestas con características de lectura global que, se espera, faciliten el contacto de pares en los temas que puedan ser comunes para los grupos de investigación.

Con respecto a nuestra sección central, como se observará en los distintos artículos, en los últimos años han proliferado las diferentes propuestas gubernamentales que incorporan la educación física, el deporte y la recreación para atender diferentes tópicos sociales, que responden a intereses estatales e internacionales. En este terreno se encuentra una variedad de programas y problemas de la más amplia procedencia. Solo algunos de ellos buscan: la selección de talentos; el desarrollo de atletas olímpicos; el financiamiento deportivo; la relación entre deporte de alto rendimiento y recreativo; la prevención de la violencia en los espectáculos deportivos; los usos y modos de apropiación de los espacios públicos, ligados a la realización de prácticas corporales, y las concepciones de cuerpo, la salud pública, la inclusión

\footnotetext{
1 Profesor y Licenciado en Educación Física por la Universidad Nacional de La Plata (UNLP). Magíster en Antropología Social por el IDES-IDAES/Universidad Nacional de San Martín. Estudiante del Doctorado con mención en Ciencias Sociales y Humanas de la Universidad Nacional de Quilmes. Becario de la Comisión de Investigaciones Científicas de la provincia de Buenos Aires. Ayudante diplomado de la cátedra de Metodología de la Investigación en Educación Física en al UNLP. Correo electrónico: levoratti@gmail.com

2 Magíster en Investigación Social Interdisciplinaria, especialista en Teorias, Métodos y Técnicas de Investigación Social y licenciado en Educación Física. Docente de tiempo completo de la Facultad de Educación Física de la Universidad Pedagógica Nacional. Editor de la revista Lúdica Pedagógica. Correo electrónico: jcarreno@pedagogica.edu.co
} 
social y educativas y su relación con: la construcción de las alteridades, la discapacidad, las adicciones a las drogas o al alcohol, la deserción escolar, la violencia juvenil, entre otros. En consecuencia, este número presenta un amplio espectro de investigaciones que, a partir de los agudos y minuciosos análisis de sus autores, posibilitan una comprensión profunda sobre estas temáticas y de la sociedad actual.

En este terreno se identifican trabajos que permiten reflexionar sobre los lineamientos que estructuran distintas propuestas gubernamentales y en otros casos se focalizan en las modalidades de intervención que promueven distintos programas y proyectos. En ambos casos, queda en primera plana la necesidad de la construcción de conocimiento crítico desde las distintas disciplinas y la educación física en particular, para la implementación de propuestas más compresivas y que motiven el cambio en esta sociedad. En todos los estudios, quedan claras las posibilidades de estas pesquisas y su necesidad en la producción de conocimiento que permita establecer políticas públicas que superen las visiones parcializadas, sesgadas, sectoriales y etnocéntricas en las que se desenvuelven gran parte de ellas. De esta manera se abre el espectro para adelantar iniciativas más inclusivas y democráticas en el campo de lo corporal.

Para ello, el lector transitará por los distintos textos compilados en esta sección central, y accederá a un amplio panorama sobre la relación de las políticas públicas destinadas al deporte, la educación física y a la recreación en cuatro países de la región. El orden de los trabajos surge de la conjunción entre las afinidades temáticas de los artículos y los campos empíricos indagados, así el lector realizará diferentes recorridos por los textos, de acuerdo con sus intereses y preocupaciones.

La sección central abre con el texto de Alejo Levoratti, una revisión de las publicaciones científicas que analizan la incorporación de la educación física, el deporte y la recreación en políticas públicas en Argentina, Brasil y Colombia en los últimos diez años. Para ello, selecciona las principales revistas de cada país y aborda los temas y problemáticas que han sido publicadas a partir de la circulación de ideas académicas; así, el autor compara las agendas científicas con el objeto de recuperar las dimensiones compartidas y singulares de cada caso.

El trabajo de Luz Elena Gallo Cadavid y Zoraida Bolívar Cartagena trata sobre la educación diferencial en las políticas pública de deporte, la recreación y la actividad física en Colombia, a partir de una reflexión profunda y propositiva sobre estas cuestiones. Las autoras pretenden mostrar que hace falta un proyecto educativo en relación con las diferencias, que ponga en "tela de juicio más la normalidad que la anormalidad". Con esta premisa, desarrollan los principios de las teorías de la educación diferencial y analizan los lineamientos que estructuran el marco legislativo de las políticas públicas y programas que se implementan en Colombia y establecen que en ellos subyace el problema de la igualdad y la diferencia. Desde su perspectiva, se enfatiza que en los procesos educativos se debe trabajar "con los otros" siendo lo central el "sentido de humanidad, el favorecimiento de las singularidades y el reconocimiento de todo ser humano como un sujeto de capacidades", hecho que exige una ética singular.

Cecilia Seré Quintero y Alexandre Fernández Vaz indagan en la construcción de lo público, el cuerpo y la política a partir del eslogan municipal, que se propone desde el Frente Amplio en 1990 hacer de "Montevideo, tu casa". Esta temática, como manifiestan los autores, tiene por objetivo analizar las relaciones entre lo público y lo privado en ocasión del borrado de sus distinciones a partir de la moderna configuración de lo 
social, de la emergencia de la economía política como forma de lectura de la realidad, y que tiene como correlato la introducción de los parámetros de la vida biológica en el espacio público.

En el trabajo de Emmanuel Ferretty se estudian las políticas de recuperación del espacio público, las cuales promueven ciertos usos corporales del espacio urbano y constituyen las condiciones de posibilidad sobre las cuales se implementan otros planes y programas deportivos, recreativos y de actividad física orientada a la salud. El autor se interroga por los sentidos, discusiones y disputas acerca de lo público; acerca de qué y cómo es el espacio público para el Estado.

Verónica Moreira estudia dos programas, "Aliento" y "Alentar", inscritos en la política deportiva de la municipalidad de Avellaneda. Este abordaje permitirá a la autora, por un lado, poner sobre la mesa cómo en las políticas deportivas de alto rendimiento se presentan particulares estructuras de práctica corporal y rendimiento de los diferentes actores, cuestión que tiene de fondo la relación entre amateurismo y profesionalismo. Por otro lado el abordaje del programa "Alentar", destinado al deporte social o recreativo, refleja la problemática de la relación entre deporte e inclusión social. El estudio de esas dos experiencias posibilitará al lector acceder a la comprensión de las circulaciones de significados y recursos financieros entre el estado nacional y municipal, al mismo tiempo que abre un campo para la discusión sobre la convivencia y relaciones entre el alto rendimiento y la recreación, en las políticas públicas destinadas al deporte.

Lino Castellani Filho analiza el proceso de mercantilización que aqueja al deporte y al tiempo libre en tiempos de megaeventos en Brasil. Tras retomar las producciones realizadas en los últimos 30 años en el campo de la educación física, efectúa una serie de críticas y propuestas para superar las políticas públicas educacionales, deportivas y de ocio de los intereses de los sectores conservadores del sector deportivo y el proceso impuesto a los países suramericanos sometidos a la lógica neoliberal.

Continuando en el escenario brasileño, Marcelo Moraes, Fernando Marinho Mezzadri, Natasha Santos, Philipe Rocha de Camargo, Katiuscia Mello Figuerôa, desde los principios teóricos de Norbert Elias, indagan en los procesos de configuración de los lineamientos que dan sentido al financiamiento deportivo de alto rendimiento y a la "Bolsa Atleta" para el desarrollo de deportistas olímpicos. A partir del análisis de la configuración de los sentidos legítimos del deporte desde los Juegos Olímpicos de Sídney 2000, y procesos de discusión y sanción de las leyes del deporte, queda al descubierto la intención de convertir al Brasil en un país con verdadero potencial olímpico.

José Garriga Zucal trata la problemática de las políticas públicas destinadas a la violencia en el fútbol en Argentina. Para esto, considera la violencia como una práctica social con sentido para los actores involucrados y su inscripción en el caso del fútbol argentino dentro de la cultura del aguante, como elemento de construcción identitaria. El autor comenta, a la vez que propone, la planificación de políticas de prevención, cuyo eje central está asociado a escudriñar en los sentidos de estas prácticas para nutrirse, abordarlos y modificarlos.

El antepenúltimo de los trabajos de la sección central es el de Federico Czesli y Diego Murzi quienes presentan una experiencia, de taller, que realizaron con alumnos de escuelas secundarias de la Ciudad Autónoma de Buenos Aires, con el objeto de reducir la violencia en el fútbol. Desde una perspectiva similar a la de Garriga Zucal sobre la 
violencia en el fútbol, los autores comparten los lineamientos y reflexiones posteriores de sus intervenciones para la planificación de políticas públicas en la materia.

La reseña de Marco Maiori, sobre el libro Políticas públicas, qualidade de vida e atividade física, de los organizadores Gutiérrez Gustavo Luis, Vilarta Roberto y Teixeira Mendes Ricardo, aporta a la sección central una temática en la cual habría un nicho vacío, como son las políticas públicas relacionadas con la educación física para la adquisición de hábitos saludables. El texto seleccionado tiene la riqueza de compilar un amplio panorama sobre más de 20 programas implementados en Brasil, que se estructura con la obtención de una buena calidad de vida.

Como observará el lector, se sugiere un recorrido amplio y heterogéneo, que no tiene como propósito cerrar las temáticas y discusiones vertidas, sino que busca abrir y contribuir a los debates desde distintas perspectivas teóricas, campos disciplinarios, propuestas gubernamentales y territorialidades. ¡Buena lectura! 